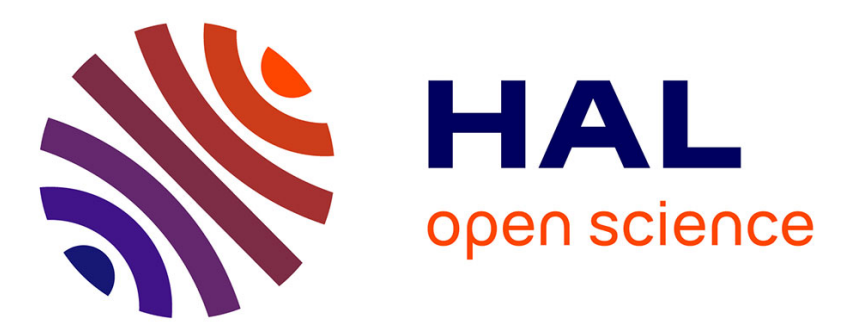

\title{
On the benefits of successive interference cancellation for ultra narrow band networks: Theory and application to IoT
}

Yuqi Mo, Claire Goursaud, Jean-Marie Gorce

\section{- To cite this version:}

Yuqi Mo, Claire Goursaud, Jean-Marie Gorce. On the benefits of successive interference cancellation for ultra narrow band networks: Theory and application to IoT. IEEE ICC 2017 - IEEE International Conference on Communications, May 2017, Paris, France. pp.1 - 6, 10.1109/ICC.2017.7996900 . hal-01610465

\section{HAL Id: hal-01610465 \\ https://hal.inria.fr/hal-01610465}

Submitted on 4 Oct 2017

HAL is a multi-disciplinary open access archive for the deposit and dissemination of scientific research documents, whether they are published or not. The documents may come from teaching and research institutions in France or abroad, or from public or private research centers.
L'archive ouverte pluridisciplinaire HAL, est destinée au dépôt et à la diffusion de documents scientifiques de niveau recherche, publiés ou non, émanant des établissements d'enseignement et de recherche français ou étrangers, des laboratoires publics ou privés. 


\title{
On the Benefits of Successive Interference Cancellation for Ultra Narrow Band Networks : Theory and Application to IoT
}

\author{
Yuqi $\mathrm{MO}^{* \dagger}$, Claire GOURSAUD* and Jean-Marie GORCE* \\ ${ }^{*}$ CITI Labs, INSA-Lyon, Villeurbanne, France \\ ${ }^{\dagger}$ Sigfox Company, Building E-volution, Labège, France \\ Email: mo.yuqi@insa-lyon.fr; claire.goursaud@insa-lyon.fr; jean-marie.gorce@insa-lyon.fr;
}

\begin{abstract}
UNB (Ultra Narrow Band) stands out as one promising PHY solution for low-power, low-throughput and long-range IoT. The dedicated MAC scheme is RFTMA (Random Frequency and Time Multiple Access), where nodes access the channel randomly both in frequency and in time domain, without prior channel sensing. This blind randomness sometimes introduces interference and packet losses. Hence, in this paper, we propose to use the well-known SIC (Successive Interference Cancellation) to cancel the interference in a recursive way. We provide a theoretical analysis of network performance, when considering jointly SIC and the specific spectral randomness of UNB. We analytically and numerically highlight the SIC efficiency in enhancing UNB system performance.
\end{abstract}

\section{INTRODUCTION}

IoT (Internet of Things) has been a hot issue in recent years, both in the research fields, and on the market. The expectation that everything is going to be connected is leading the trend. But, as more and more devices are being deployed in cities and rural areas, high communication capabilities are demanded for IoT networks, as well as the scalability to allow huge coverage [1].

The applications of IoT are very diverse : from indoor to outdoor, from smart meter's several bytes of data per day to vehicle monitor's huge amount of information per milliseconds, and based on bluetooth, WiFi to sub-GHz [2]. With different features, their particular requirements vary.

However, most of the devices do not have ultimate demands. They only have a small amount of data to transfer, and do not require a response within a milliseconds delay. Besides, while IoT devices are mostly battery-based, it would be difficult to often change batteries of these numerous devices. Therefore, the challenges for IoT networks are to achieve high scalability to handle massive nodes, wide coverage, while keeping low energy consumption, and low node cost.

Devices with this kind of demands are difficult to be integrated into traditional cellular networks due to their sporadic activities. That's why recent technologies have come to the scene dedicated for LPWAN (Low Power Wide Area Network) [3]. The first one is NB-IoT, which has been included to the 3GPP standardization, and is the narrow band system based on LTE [4]. Besides, CSS (commercially known as LoRa) [5] and RPMA (developed by Ingenu) [6] are both based on spread spectrum, in unlicensed ISM bands. Finally, UNB
(Ultra Narrow Band), which was developed and is deployed by SigFox, also in sub-GHz unlicensed band [7]. UNB permits longer transmission range than spread spectrum, and it is expected to be more energy efficient [3]. Hence, we focus, in this paper, on the use of UNB.

In a UNB system, the signal used to transfer information occupies a very small band compared to classical systems, typically $100 \mathrm{~Hz}$. This permits to have long transmission coverage (up to $50 \mathrm{~km}$ in practice), ultra low power consumption, and it is believed to be perfect for devices which have small size of messages, essentially 100 up to 200 bits [3].

The main specificity of UNB is linked to the oscillator drift, which is a typical phenomenon in electronic devices. It characterizes the fact that the actual generated carrier frequency is not exactly the expected one. This imprecision comes from oscillators factoring. To the best of our knowledge, the most precise oscillator $(0.25 \mathrm{ppm}$ drift) still leads to an inaccuracy of $217 \mathrm{~Hz}$ for a targeted frequency at $868 \mathrm{MHz}$. This uncertainty is larger than the individual UNB signal bandwidth. As drift is inevitable in UNB system, channelization would lead to the waste of frequency resources [10].

As a consequence, a specific dedicated MAC is considered. This channel access scheme is named RFTMA (Random Frequency and Time Multiple Access) [8]. Each node sends messages at any moment and at a carrier frequency randomly chosen at its will, without previously analyzing the channel state. The advantage of this approach, is that the classical overhead dedicated to the reservation of radio resources is saved. Nonetheless, as there is no control, collisions may occur. This interference potentially generated by simultaneous transmissions needs to be avoided in UNB networks [9].

As the interference is not controlled at the transmitter side, for cost and energy consumption reasons, we focus on the interference mitigation at the BS (Base Station) side. Among the IC (Interference Cancellation) technologies, we can first cite PIC (Parallel Interference Cancellation). PIC processes simultaneously all the users and cancels their interference after they have all been independently decoded. However, PIC is considered unprofitable for massive practical implementations, as it demands precious hardwares [13].

SIC (Successive Interference Cancellation) is another IC technology, which attempts to remove the interference in a 
recursive way [11][14], by exploiting the diversity of the received signal strengths. SIC is expected to be the most efficient IC-based methodology in terms of Bit-Error-Rate performance. Nevertheless, as the accuracy and robustness demand increases, the complexity of iterative detection and decoding process grows too. Hence there is a trade-off between the performance of SIC and the complexity [12]. SIC was proved to be highly beneficial when low-rate codes are used [15]. We thus consider SIC in this study.

More recently, [16] has considered SIC receiver to improve IoT networks. A normalized theoretical analysis of the capture probability by considering the MPR (Multiple Packet Reception) and SIC is presented. Different channel models, such as path loss, general fading and shadowing are considered. This study confirms the adequacy of the SIC to IoT. However, the interference model refers only to the aggregated power of interferers' contribution when the same channel is used. In a UNB system, as nodes select their frequency randomly in a continuous space, overlaps of signals can generate interference with an additional degree of freedom. Indeed, in UNB network, the interference also depends on the frequency spacing of potential interferers. For this reason, we can not directly derive the UNB performances from this generalized analysis. Thus, in this paper, we propose to analyze the SIC benefits in a UNB network. We exploit the results presented in [17], which characterizes analytically the performance of UNB with a simple receiver when considering both path loss and the spectrum random access.

The rest of this paper is organized as follows : Section II gives the modeling and hypothesis. Section III provides the theoretical analysis of the performance without and with SIC. The numerical results and the validation of theory are given in Section IV, then Section V concludes.

\section{Modeling And Assumptions}

We consider a network with a single BS located at the cell center. The BS is supposed to be constantly in reception state. Nodes (devices) are positioned randomly and uniformly in a disk area, defined by the radius $\left[r_{m}, r_{M}\right] . r_{m}$ corresponds to an exclusion area around the BS where no node is deployed. Thus, free space propagation model can be used without having the path loss being $\infty$ when $r$ tends to 0 .

We suppose that all nodes have the same behavior : they send their message packets of the same size, with the same emission power and antenna gain, and they have the same wake-up duty cycle. Nonetheless, as nodes have various positions, and path loss is considered, their received power at the BS differs.

The specificity of UNB systems is that the channel access scheme is totally random. Nodes select randomly and independently their carrier frequency. Hence, interference may occur as there is no control and may lead to transmission errors. The criteria chosen to measure the network performance is the PER (Packet Error Rate), which depends on the SIR (Signal to Interference Ratio). When the SIR of a packet is lower than the predefined threshold $S$, the packet is considered lost.
We theoretically derive the performance by observing the network state during a given instant. We define $N$ as the number of active nodes at the observed moment. We elect one as the desired node, while all the other nodes are potential interfering nodes. The interference level required to generate a packet loss can be due to a single interferer, or to the aggregation of several interferers' contribution. However, one may note that collisions occur most often between only 2 nodes at a time [8]. Indeed, in a UNB based network, as the individual signal bandwidth is very narrow compared to the whole available bandwidth, more than one interferer rarely happens. Thus, we focus on the single interferer case.

We suppose that the desired node transmits at frequency $f_{1}$, and the potential interfering node at frequency $f_{2}$. The main parameter is their frequency spacing $\Delta f=f_{1}-f_{2}$, which determines the interference contribution of each interfering node. When two nodes choose their frequency close enough, interference generated would cause packet losses.

We have used the interference model described in [8]. According to realistic UNB network parameters and the used filter, the interference power density can be approximated by a zero-mean Gaussian function, depending on $\Delta f$ :

$$
\beta(\Delta f)=\frac{150}{\sigma \sqrt{2 \pi}} \exp \frac{-\Delta f^{2}}{2 \sigma^{2}}
$$

with $\sigma=60$ for a $100 \mathrm{bit} / \mathrm{s}$ transmission.

\section{TheORETICAL ANALYSiS}

\section{A. Preliminary: Derivation of PER without SIC}

In [17], we have derived the PER for a simple BPSK receiver, when considering both the spectral randomness and path loss. We report here the main derivation steps as a basis for adapting to the SIC case III-B.

We consider the case where there are only two nodes (node 1 as the desired node and node 2 as the interfering one) at the observed moment. The PER of the desired node 1 can be written as:

$$
P E R=P\left(S I R_{1} \leq S\right)
$$

We suppose that node 1 (resp. node 2) is at a distance of $r_{1}$ (resp. $r_{2}$ ). $P_{0}$ is the signal power at the reference distance of $r_{0}$. With a free space propagation model, the $S I R$ of the desired node is :

$$
S I R_{1}=\frac{P_{0}\left(\frac{r_{0}}{r_{1}}\right)^{2}}{P_{0}\left(\frac{r_{0}}{r_{2}}\right)^{2} \beta(\Delta f)}=\left(\frac{r_{2}}{r_{1}}\right)^{2} \frac{1}{\beta(\Delta f)}
$$

Consequently, (2) can also be expressed as:

$$
P E R=P\left(r_{2} \leq r_{1} \sqrt{S \beta(\Delta f)}\right)
$$

According to the law of total probability, we split (4) depending on the value of $\sqrt{S \beta(\Delta f)}$, conditionally to $\Delta f$. We obtain an expression depending on several parameters : the total available bandwidth $B$, the $S I R$ threshold $S$, and the range of the area $\left[r_{m}, r_{M}\right]$ : 


$$
\begin{aligned}
P E R= & \int_{0}^{B} P\left(r_{2} \leq r_{1} \sqrt{S \beta(\Delta f)} \mid \Delta f\right) P(\Delta f) \mathrm{d} \Delta f \\
= & \int_{b_{2}}^{b_{1}}\left(\frac{a}{S \beta(\Delta f)}+b S \beta(\Delta f)+c\right) P(\Delta f) \mathrm{d} \Delta f \\
& +\int_{b_{3}}^{b_{2}}\left(\frac{d}{S \beta(\Delta f)}+e S \beta(\Delta f)+f\right) P(\Delta f) \mathrm{d} \Delta f \\
& +\int_{b_{4}}^{b_{3}} 1 * P(\Delta f) \mathrm{d} \Delta f
\end{aligned}
$$

with the following constants:

$$
\begin{aligned}
a & =\frac{r_{m}{ }^{4}}{2 k^{4}}, & d=\frac{r_{M}^{4}}{2 k^{4}}-\frac{r_{m}^{2} r_{M}^{2}}{k^{4}}-\frac{r_{M}^{2}}{k^{2}} \\
b & =\frac{r_{M}{ }^{4}}{2 k^{4}}, & e=-\frac{r_{m}^{4}}{2 k^{4}} \\
c & =-\frac{r_{M}^{2} r_{m}^{2}}{k^{4}}, & f=\frac{r_{m}^{4}}{k^{4}}+\frac{r_{M}^{2}}{k^{2}}
\end{aligned}
$$

where $k^{2}=r_{M}^{2}-r_{m}^{2}$;

and with the following integral edges: $b_{1}=$ $\min \left(\beta^{-1}\left(\left(\frac{r_{m}}{r_{M}}\right)^{2} \frac{1}{S}\right), B\right), b_{2}=\min \left(\beta^{-1}\left(\frac{1}{S}\right), B\right)$ $b_{3}=\min \left(\beta^{-1}\left(\left(\frac{r_{M}}{r_{m}}\right)^{2} \frac{1}{S}\right), B\right)$, and $b_{4}=0$;

and where $P(\Delta f)$ which represents the probability distribution function of $\Delta f=\left|f_{2}-f_{1}\right|$. As the carrier frequency of the two nodes are randomly and independently chosen in $[0, B]$, we have:

$$
P(\Delta f)= \begin{cases}\frac{2}{B}\left(1-\frac{\Delta f}{B}\right) & \text { for } \Delta f \in[0, B] \\ 0 & \text { elsewhere }\end{cases}
$$

The final expression of PER without SIC (5) will be explored and extended to derive the PER with SIC in the following part.

\section{B. Derivation of PER with SIC}

In this section, we consider that the BS uses a SIC receiver to extract more packets. The network behavior assumptions remain the same.

The principle of SIC is to successively decode packets contained in the received signal. Suppose that the BS simultaneously receives, for example $l$ packets, which are all in collision. Without SIC, only the packet having the highest SIR (or received power, depending on the measure criteria) can be correctly decoded, as long as its SIR is above the required threshold. Meanwhile, with SIC, the knowledge on this packet can be exploited to reconstruct the interfering signal and subtract it from the total received signals. The BS then performs the decoding on the remaining $l-1$ packets. The process goes on until the packet with the highest SIR doesn't fulfill the criteria for successful decoding.

We evaluate the SIC performance by focusing on the one iteration case, as we make the assumption that there is only one interferer. The case where a collision caused by the aggregation of several interferers is still neglected.

We further suppose that the SIC receiver can perfectly reconstruct decoded signals, and subtract them from the received signals. Hence, when two nodes are interfering each other, once one node is successfully decoded, the other one will also be decoded with the SIC receiver.

To derive the packet success probability, we identify two scenarios where the packet transmitted by the node 1 is decoded: when node 1 is directly decoded by the BS, as its SIR is high enough; or when node 1 is not decoded in the first place, but the interfering node is: thanks to the SIC receiver, node 1 can then be decoded. Hence the success probability of the desired node can be expressed as:

$$
P_{s}=P\left(S I R_{1}>S\right)+P\left(S I R_{1} \leq S \cap S I R_{2}>S\right)
$$

As the SIR of node 1 and node 2 depend on the same frequency difference $\Delta f$, their related probabilities are correlated. Therefore, we can not treat $P\left(S I R_{1} \leq S \cap S I R_{2}>S\right)$ independently.

Thus the PER becomes:

$$
\begin{aligned}
P E R_{S I C} & =1-P_{s} \\
& =1-\left(P\left(S I R_{1}>S\right)+P\left(S I R_{1} \leq S \cap S I R_{2}>S\right)\right) \\
& =P\left(S I R_{1} \leq S\right)-P\left(S I R_{1} \leq S \cap S I R_{2}>S\right)
\end{aligned}
$$

The first part $\left(P\left(S I R_{1} \leq S\right)\right)$ is already available in Section III-A. We derive $P\left(S I R_{1} \leq S \cap S I R_{2}>S\right)$ in the following part.

$$
\begin{aligned}
& P\left(S I R_{1} \leq S \cap S I R_{2}>S\right) \\
& =\int_{0}^{B} P\left(r_{2} \leq r_{1} \sqrt{S \beta(\Delta f)} \cap r_{2}<\frac{r_{1}}{\sqrt{S \beta(\Delta f)}} \mid \Delta f\right) P(\Delta f) \mathrm{d} \Delta f
\end{aligned}
$$

We use a similar method as in [17] to derive this PER. We compute the inner part of the integral by observing that depending on the value of $\sqrt{S \beta(\Delta f)}$, the intersection of the two inequalities reduces to 2 cases:

$$
\begin{aligned}
& P\left(r_{2} \leq r_{1} \sqrt{S \beta(\Delta f)} \cap r_{2}<\frac{r_{1}}{\sqrt{S \beta(\Delta f)}} \mid \Delta f\right) \\
& = \begin{cases}P\left(r_{2} \leq r_{1} \sqrt{S \beta(\Delta f)} \mid \Delta f\right) & \text { if } \sqrt{S \beta(\Delta f)} \leq 1, \\
P\left(r_{2}<\frac{r_{1}}{\sqrt{S \beta(\Delta f)}} \mid \Delta f\right) & \text { if } \sqrt{S \beta(\Delta f)} \geq 1\end{cases}
\end{aligned}
$$

We now evaluate the probabilities (12) separately for each case in the following parts.

1) $\sqrt{S \beta(\Delta f)} \leq 1$ : It is related to the first line in (12). In this case, the interfering node is always closer to the receiver than the desired node. This probability is computed by evaluating all cases for $r_{1}$ :

$$
\begin{aligned}
& P\left(r_{2} \leq r_{1} \sqrt{S \beta(\Delta f)} \mid \Delta f\right) \\
& = \begin{cases}0 & \text { if } \sqrt{S \beta(\Delta f)} \leq \frac{r_{m}}{r_{M}} \\
\int_{\frac{r_{m}}{\sqrt{S \beta(\Delta f)}} \frac{r_{1}{ }^{2} S \beta(\Delta f)-r_{m}^{2}}{r^{2}} P\left(r_{1}\right) \mathrm{d} r_{1}} & \text { if } \frac{r_{m}}{r_{M}} \leq \sqrt{S \beta(\Delta f)} \leq 1\end{cases} \\
& = \begin{cases}0 & \text { if } \sqrt{S \beta(\Delta f)} \leq \frac{r_{m}}{r_{M}} \\
\frac{a}{S \beta(\Delta f)}+b S \beta(\Delta f)+c & \text { if } \frac{r_{m}}{r_{M}} \leq \sqrt{S \beta(\Delta f)} \leq 1\end{cases}
\end{aligned}
$$




$$
\begin{aligned}
P E R_{S I C}= & \frac{2}{B}\left[\frac{\left(d-d_{1}\right) \sigma^{2} \pi}{S 150} \operatorname{erfi}\left(\frac{\Delta f}{\sqrt{2 \sigma^{2}}}\right)+75\left(e-e_{1}\right) S \operatorname{erf}\left(\frac{\Delta f}{\sqrt{2 \sigma^{2}}}\right)+\left(f-f_{1}\right) \Delta f-\frac{\left(d-d_{1}\right) \sigma^{3} \sqrt{2 \pi}}{B S 150} \exp \frac{\Delta f^{2}}{2 \sigma^{2}}+\frac{\left(e-e_{1}\right) S 150 \sigma}{B \sqrt{2 \pi} \exp \frac{\Delta f^{2}}{2 \sigma^{2}}}-\frac{\left(f-f_{1}\right)(\Delta f)^{2}}{2 B}\right]_{b_{3}}^{b_{2}} \\
& +\frac{2}{B}\left[\Delta f-\frac{\Delta f^{2}}{2 B}\right]_{b_{4}}^{b_{3}}
\end{aligned}
$$

with the same constants $a, b, c$ as the no-SIC case (5).

$P(r)$ represents the probability that any node in a disk form area of $\left[r_{m}, r_{M}\right]$ is located at a distance $r$ from the BS. As nodes positions are distributed uniformly and randomly, we have:

$$
P(r)= \begin{cases}\frac{2 r}{r_{M}^{2}-r_{m}^{2}}=\frac{2 r}{k^{2}} & \text { for } r \in\left[r_{m}, r_{M}\right] \\ 0 & \text { elsewhere }\end{cases}
$$

with $k^{2}=r_{M}^{2}-r_{m}^{2}$.

2) $\sqrt{S \beta(\Delta f)} \geq 1$ : This is related to the second case of (12). It indicates that the interfering node can be further from the receiver than the desired node. We also decompose it conditionally to $r_{1}$.

$$
\begin{aligned}
& P\left(r_{2}<\frac{r_{1}}{\sqrt{S \beta(\Delta f)}} \mid \Delta f\right) \\
& =\int_{r_{m}}^{r_{M}} P\left(r_{2}<\frac{r_{1}}{\sqrt{S \beta(\Delta f)}} \mid \Delta f \cap r_{1}\right) P\left(r_{1}\right) \mathrm{d} r_{1}
\end{aligned}
$$

Hence we can compute the probability by the condition of both $\Delta f$ and $r_{1}$, that $r_{2}$ is smaller that a specific value, in the considered range $\left[r_{m}, r_{M}\right]$ :

$$
\begin{aligned}
& P\left(r_{2}<\frac{r_{1}}{\sqrt{S \beta(\Delta f)}} \mid \Delta f \cap r_{1}\right) \\
& = \begin{cases}0 & \text { if } \frac{r_{1}}{\sqrt{S \beta(\Delta f)}} \leq r_{m}, \\
\int_{r_{m}} \frac{r_{1}}{\sqrt{S \beta(\Delta f)}} P\left(r_{2}\right) \mathrm{d} r_{2} & \text { if } r_{m} \leq \frac{r_{1}}{\sqrt{S \beta(\Delta f)}} \leq r_{M} \\
0 & \text { if } \frac{r_{1}}{\sqrt{S \beta(\Delta f)}} \geq r_{M}\end{cases} \\
& = \begin{cases}0 & \text { if } \frac{r_{1}}{\sqrt{S \beta(\Delta f)}} \leq r_{m}, \\
\frac{1}{k^{2}} \frac{r_{1}^{2}}{S \beta(\Delta f)}-\frac{r_{m}^{2}}{k^{2}} & \text { if } r_{m} \leq \frac{r_{1}}{\sqrt{S \beta(\Delta f)}} \leq r_{M} \\
0 & \text { if } \frac{r_{1}}{\sqrt{S \beta(\Delta f)}} \geq r_{M}\end{cases}
\end{aligned}
$$

The first part is null because the condition implies that node 2 enters the exclusive zone (where the radius is smaller than $r_{m}$ ). As no nodes can be in this zone, the probability is zero. The second part corresponds to the fact that the interfering node is in the area range, but the integral upper limit depends on the location of the desired node. As $\sqrt{S \beta(\Delta f)} \geq 1$, the third condition of (19) indicates that $r_{1}$ is larger that $r_{M}$. Meanwhile, as $r_{1}$ is constrained by $\left[r_{m}, r_{M}\right]$, thus it leads to a null probability.
From (19), we can deduce the expression of (16) as below:

$$
\begin{aligned}
& P\left(r_{2}<\frac{r_{1}}{\sqrt{S \beta(\Delta f)}} \mid \Delta f\right) \\
& = \begin{cases}\int_{r_{m} \sqrt{S \beta(\Delta f)}}^{r_{M}}\left(\frac{1}{k^{2}} \frac{r_{1}^{2}}{S \beta(\Delta f)}-\frac{r_{m}^{2}}{k^{2}}\right) \frac{2 r_{1}}{k^{2}} \mathrm{~d} r_{1} & \text { if } 1 \leq \sqrt{S \beta(\Delta f)} \leq \frac{r_{M}}{r_{m}} \\
0 & \text { if } \sqrt{S \beta(\Delta f)} \geq \frac{r_{M}}{r_{m}}\end{cases} \\
& = \begin{cases}\frac{d_{1}}{S \beta(\Delta f)}+e_{1} S \beta(\Delta f)+f_{1} & \text { if } 1 \leq \sqrt{S \beta(\Delta f)} \leq \frac{r_{M}}{r_{m}} \\
0 & \text { if } \sqrt{S \beta(\Delta f)} \geq \frac{r_{M}}{r_{m}}\end{cases}
\end{aligned}
$$

with the following constants:

$$
d_{1}=\frac{r_{M}^{4}}{2 k^{4}}, \quad e_{1}=\frac{r_{m}{ }^{4}}{2 k^{4}}, \quad f_{1}=-\frac{r_{M}{ }^{2} r_{m}{ }^{2}}{k^{4}}
$$

3) Final Expression: As we have the results of both two cases, (14) and (21), we can then derive:

$$
\begin{aligned}
& P\left(S I R_{1} \leq S \cap S I R_{2}>S\right) \\
& =\int_{0}^{B} P\left(r_{2} \leq r_{1} \sqrt{S \beta(\Delta f)} \cap r_{2}<\frac{r_{1}}{\sqrt{S \beta(\Delta f)}} \mid \Delta f\right) P(\Delta f) \mathrm{d} \Delta f \\
& =\int_{b_{2}}^{b_{1}}\left(\frac{a}{S I R_{t h} \beta(\Delta f)}+b S I R_{t h} \beta(\Delta f)+c\right) P(\Delta f) \mathrm{d} \Delta f \\
& \quad+\int_{b_{3}}^{b_{2}}\left(\frac{d_{1}}{S I R_{t h} \beta(\Delta f)}+e_{1} S I R_{t h} \beta(\Delta f)+f_{1}\right) P(\Delta f) \mathrm{d} \Delta f
\end{aligned}
$$

The integral edges $b_{1}, b_{2}, b_{3}$ are the same than the PER without SIC (5).

Therefore, the PER of the SIC receiver becomes :

$$
\begin{aligned}
P E R_{S I C}= & P\left(S I R_{1} \leq S\right)-P\left(S I R_{1} \leq S \cap S I R_{2}>S\right) \\
= & \int_{b_{3}}^{b_{2}}\left(\frac{\left(d-d_{1}\right)}{S I R_{t h} \beta(\Delta f)}+\left(e-e_{1}\right) S I R_{t h} \beta(\Delta f)+\left(f-f_{1}\right)\right) P(\Delta f) \mathrm{d} \Delta f \\
& +\int_{b_{4}}^{b_{3}} 1 * P(\Delta f) \mathrm{d} \Delta f
\end{aligned}
$$

The result of integrals is showed as (27), for the 2 users case.

Finally, we can now extend (27) to the $N$ users case. We observe one desired node, with $N-1$ potential interfering nodes at the observed moment. Accordingly, the success probability of the desired node, is that none of these $N-1$ nodes interrupts it. Therefore the PER with SIC is given by:

$$
P E R_{S I C(N)}=1-\left(1-P E R_{S I C}\right)^{N-1}
$$




\section{VALIDATION AND NUMERICAL RESULTS}

In this section we show the comparison of theoretical and simulation results, in order to validate the analytic expression of PER with SIC, as well as to demonstrate the impacts of SIC.

\section{A. Validation}

In order to validate $P E R_{S I C(N)}$, Monte Carlo simulations based on realistic network parameters have been carried out. We consider a single BS, with nodes randomly deployed with a spatial Poisson process in the BS coverage. We have considered 4 main network parameters: the number of active nodes $N$ at the considered moment, the total available bandwidth $B$, the threshold of SIR $S$, and the range of node area $\left[r_{m}, r_{M}\right]$.

Firstly, we have evaluated the variation in the decoded packet percentages against different SIC iterations, as depicted in Fig.1. The iteration 0 corresponds to the decoding process of the simple receiver. The interference cancellation part of the SIC receiver starts with iteration 1. In this figure, no limitation is imposed on the SIC : decoding is performed until no additional node can be decoded. We notice that when we raise the number of active nodes, the required SIC iterations increase as well. Nonetheless, iteration 1 brings the most significant performance improvement in all cases. Therefore, these results confirm that one iteration is accurate to evaluate the SIC performances, as was supposed in Section III-B.

Fig.2-3 present the performance of the simple receiver and the SIC receiver, both by simulation and theory. We can observe that simulations (points) coincide perfectly with the theory (lines). We obtain the same results when varying $B$ and the area range, which are not presented here due to space constraint. Therefore, we can conclude that the theoretical expression (28) is validated.

Besides, we verify the classical behavior of the PER when varying the different parameters. Indeed, as the number of active nodes $N$ in a certain bandwidth grows, or as the threshold of SIR $S$ increases, the PER decreases. The choice of $S$ is predefined according to the demanded QoS. These phenomena reveal that when nodes are too dense for the total available bandwidth, or when the condition of success becomes too strict, the network performance degrades.

\section{B. Analysis of SIC performance}

We analyze the impact of SIC in terms of performance improvement in this part. As demonstrated in Fig.2-3, the packet error rate is always smaller with the SIC receiver. Accordingly, we can conclude that SIC is beneficial in mitigating the interference induced by the random spectrum access.

To further evaluate the SIC improvement, we define the gain of SIC as $\frac{P E R_{n o S I C}-P E R_{S I C}}{P E R_{\text {noSIC }}}$, which is the percentage of error reduction thanks to SIC. We use it as an indicator of SIC efficiency. Besides, for the sake of generality, we characterize the network activity by the normalized node density, which is the total active nodes' spectrum occupation over the total bandwidth. Typically one signal occupies $100 \mathrm{~Hz}$ spectrum in $\mathrm{UNB}$, thus the normalized node density is $\frac{100 N}{B}$.

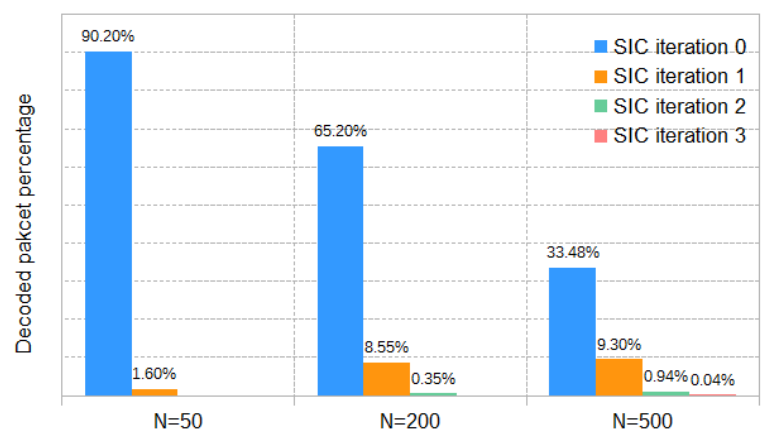

Fig. 1. Decoded packet percentage vs different SIC iterations, for different active node numbers, $B=96 \mathrm{kHz}, S=6.8 \mathrm{~dB}, r_{m}=30 \mathrm{~m}, r_{M}=1000$ $\mathrm{m}$.

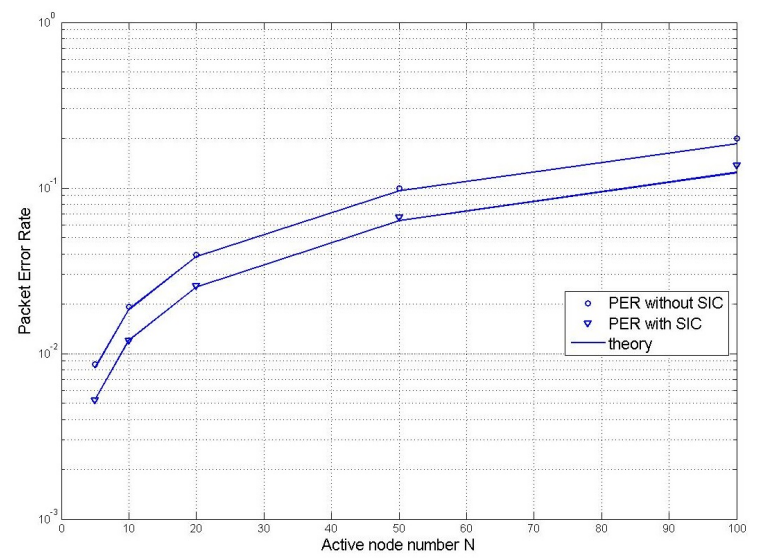

Fig. 2. PER without SIC vs with SIC, for different active node numbers $N$, $B=96 \mathrm{kHz}, r_{m}=30 \mathrm{~m}, r_{M}=1000 \mathrm{~m}, S=6.8 \mathrm{~dB}$.

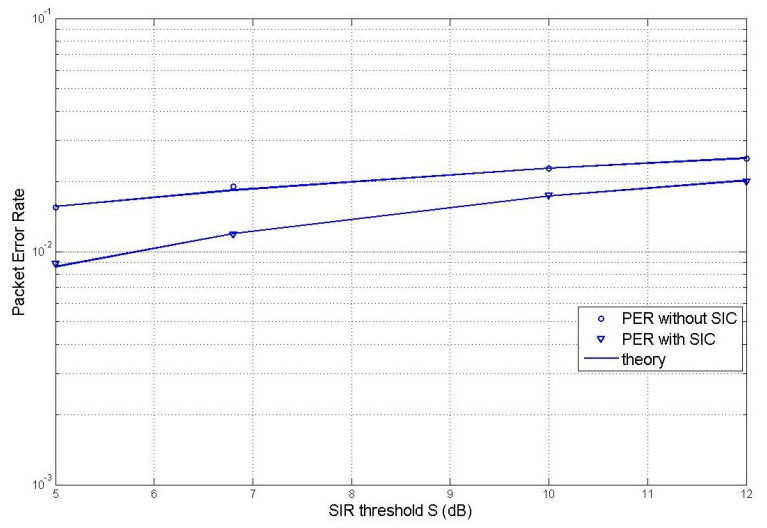

Fig. 3. PER without SIC vs with SIC, for different SIR threshold $S(\mathrm{~dB})$, $B=96 \mathrm{kHz}, N=10, r_{m}=30 \mathrm{~m}, r_{M}=1000 \mathrm{~m}$.

We have evaluated the gain of SIC by maintaining the node density at a constant level, as demonstrated in Fig.4. The idea is to test whether the SIC gain changes for different scales of node number, while keeping the node density fixed. We observe that no matter how the scale of node number becomes, as long as the node density is constant, their SIC improvement is identical. Furthermore, we verify the evolution of SIC gain 


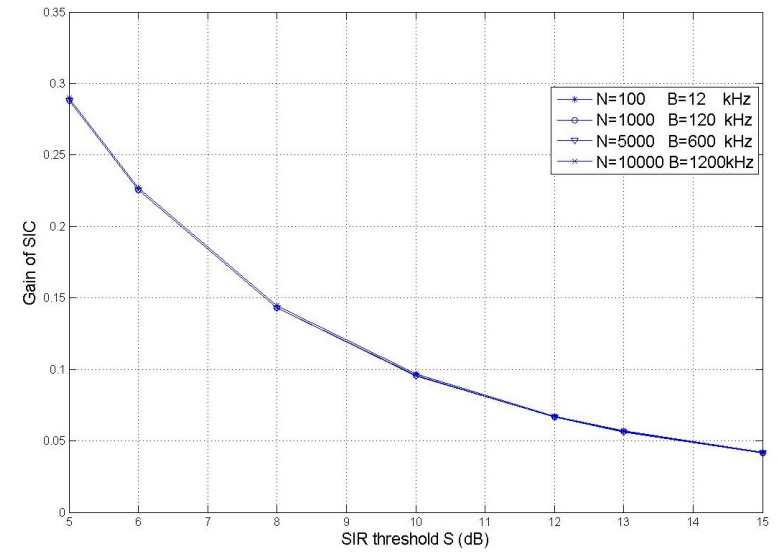

Fig. 4. Gain of SIC, for different SIR threshold $S(\mathrm{~dB})$, and constant node density $\frac{100 N}{B}, r_{m}=30 \mathrm{~m}, r_{M}=1000 \mathrm{~m}$.

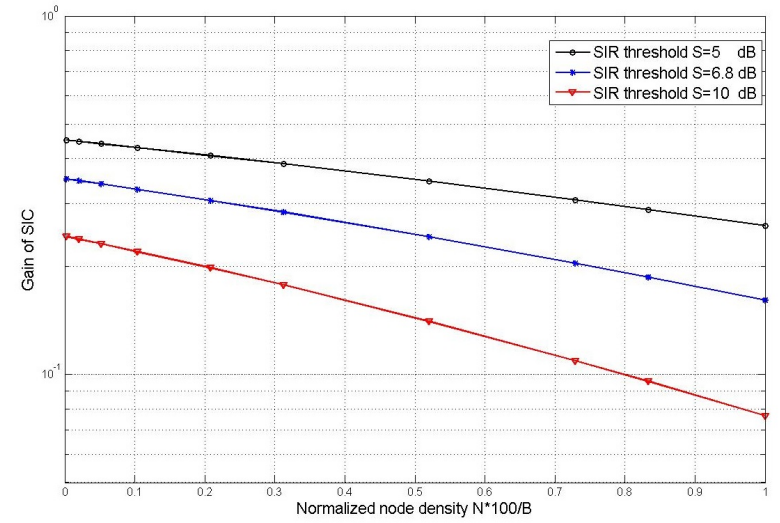

Fig. 5. Gain of SIC, for different node density $\frac{100 N}{B}$, and different SIR threshold $S(\mathrm{~dB}), r_{m}=30 \mathrm{~m}, r_{M}=1000 \mathrm{~m}$.

when the node density is not constant. As shown in Fig. 5, we see that the SIC gain degrades as the node density increases. And for the same node density, the higher $S$ is, the lower the SIC gain becomes.

Such results can be directly exploited to simply adapt the network parameters to the targeted load. For instance, for a given node number, we can adapt the bandwidth to obtain the expected SIC gain. Furthermore, these results also permit to foresee the SIC performance for different node densities.

\section{CONCLUSION}

In this paper, we have considered UNB based IoT networks, in which the channel access is totally random both in time and frequency domains. Interference is thus a main limitation of the system. Therefore, we have proposed to apply the SIC methodology to the UNB system (where interference has a specific behavior), in order to mitigate the interference impact. We have derived the theoretical expression of PER with SIC, by considering the specific random spectrum access of UNB. We have validated the theoretical expression with simulations. According to the analytic and numerical results, SIC has effectively reduced the probability of errors in UNB system. We have highlighted that the increase of node density would cause the degradation of SIC performance; and that for the same node density, the SIC efficiency for enhancing the network performance maintains constant.

\section{ACKNOWLEDGMENT}

The authors would like to thank Christophe Fourtet (Sigfox company) for providing realistic UNB network parameters in this study.

\section{REFERENCES}

[1] J. Gubbi, R. Buyya, S. Marusic, M. Palaniswami, "Internet of Things (IoT): A vision, architectural elements, and future directions", in Future Generation Computer Systems, Volume 29, Issue 7, September 2013, Pages 1645-1660.

[2] L. Da Xu, W. He, S. Li, "Internet of Things in industries: A survey", in IEEE Transactions on Industrial Informatics, 10(4), 2014, pp2233-2243.

[3] C. Goursaud and J. M. Gorce, "Dedicated networks for IoT: PHY / MAC state of the art and challenges", in EAI Endorsed Transactions on Internet of Things, vol. 15, no. 1, October 2015

[4] R. Ratasuk, B. Vejlgaard, N. Mangalvedhe and A. Ghosh, "NB-IoT system for M2M communication, in 2016 IEEE Wireless Communications and Networking Conference, Doha, Qatar, 2016, pp. 1-5.

[5] http://lora-alliance.org/What-Is-LoRa/Technology (accessed 2016/28/09)

[6] http://www.ingenu.com/ (accessed 2016/28/09)

[7] http://www.sigfox.com/en/\#!/technology (accessed 2016/28/09)

[8] M.-T. Do, C. Goursaud, and J.-M. Gorce, "Interference modelling and analysis of random fdma schemes in ultra narrow band networks", in The Tenth Advanced International Conference on Telecommunications, AICT 2014, pp. 132-137, July 2014.

[9] M.-T. Do, C. Goursaud, and J.-M. Gorce, "On the benefits of RandomFDMA schemes in ultra narrow band networks", in 2014 12th International Symposium on Modeling and Optimization in Mobile, Ad Hoc, and Wireless Networks (WiOpt), pp. 672-677, 12-16 May 2014.

[10] C. Goursaud, and Y. Mo "Random Unslotted Time-Frequency ALOHA: Theory and Application to IoT UNB Networks", in 23^rd International Conference on Telecommunications (ICT), 2016, 16-18 May 2016.

[11] R. Liu, Y. Shi, K. Lui, M. Sheng, Y. Wang and Y. Li, "Bandwidth-Aware High-Throughput Routing With Successive Interference Cancelation in Multihop Wireless Networks", in Vehicular Technology IEEE Transactions, vol. 64, pp. 5866-5877, 2015, ISSN 0018-9545.

[12] Miridakis, Nikolaos I., and Dimitrios D. Vergados. "A survey on the successive interference cancellation performance for single-antenna and multiple-antenna OFDM systems", in IEEE Communications Surveys and Tutorials, 15.1 (2013): 312-335.

[13] J.G. Andrews, Interference cancellation for cellular systems: a contemporary overview, in IEEE Wireless Commun., vol. 12, no. 2, pp. 19-29, 2005.

[14] Sen, Souvik, Santhapuri, Naveen, Choudhury, Romit Roy, et al. "Successive interference cancellation: A back-of-the-envelope perspective", in Proceedings of the 9th ACM SIGCOMM Workshop on Hot Topics in Networks. ACM, 2010. p. 17.

[15] X. Zhang, et M. Haenggi. "The performance of successive interference cancellation in random wireless networks", in IEEE Transactions on Information Theory, 2014, vol. 60, no 10, p. 6368-6388.

[16] Zanella, Andrea, and M. Zorzi. "Theoretical analysis of the capture probability in wireless systems with multiple packet reception capabilities", in IEEE Transactions on Communications, 60.4 (2012): 1058-1071.

[17] Y. Mo, C. Goursaud, and J.-M. Gorce,'Theoretical Analysis of UNBbased IoT Networks with Path Loss and Random Spectrum Access", in 2016 IEEE 27th Annual International Symposium on Personal, Indoor, and Mobile Radio Communications (PIMRC), 4-9 Sep. 2016. 